\title{
Enhancing a Control Systems Design Course by Using Experiential Learning Model
}

\section{Dr. Zahrasadat Alavi, California State University, Chico}

Dr. Zahrasadat Alavi, an Assistant Professor at the Department of Electrical and Computer Engineering at California State University Chico, received her PhD in Electrical Engineering from University of Wisconsin Milwaukee in May 2015. She received her B.Sc. and M.Sc. from Amirkabir University (Polytechnic of Tehran) with honors in 2007 and 2009 respectively, and another Master of Science from University of Wisconsin Milwaukee (UWM) in Electrical Engineering in 2012. She was an Assistant Professor at the Electrical and Instrumentation Department of Los Medanos College during 2016-2017 academic year. She was an Adjunct Faculty at San Francisco State University and Diablo Valley College during 2015-2016 academic year, and an instructor at UWM from January 2014 until May 2015. She has taught Control Systems Design course several times, and has adapted different methods of teaching in her classes. She is a member of IEEE, and has several publications in IEEE, ASEE and peer reviewed journals. Her primary research interests include engineering education, advanced control systems, and simulation of linear and nonlinear systems. She also conducts research in the area of digital image processing and analysis and was awarded an NSF-MRI grant to establish an FTIR Spectroscopic Imaging Lab at CSU Chico .

\section{Dr. Kathleen Meehan, California State University, Chico}

Kathleen Meehan earned her B.S. in electrical engineering from Manhattan College and her M.S. and Ph.D. from the University of Illinois. After graduation, she worked at Lytel, Inc., Polaroid Corporation, and Biocontrol Technology. She moved into academia full-time in 1997 and worked at the University of Denver, West Virginia University, and Virginia Tech. From 2013 to 2017, she was the director of the Electronics and Electrical Engineering program at University of Glasgow-University of Electronic Science and Technology of China. Dr. Meehan became chair of the Electrical and Computer Engineering Department at the California State University, Chico in 2017. She is actively involved in the development of mobile hands-on pedagogy as well as research on other topics in STEM education, the synthesis and characterization of nanoscale materials, and fermentation processes. 


\section{Enhancing Control Systems Design Course by Using Experiential Learning Model}

In this paper, authors present the outcomes of implementing an experiential learning model to explore innovative teaching pedagogy in CSU Chico EECE 482 Control Systems Design course. To reach this goal, multiple projects and demonstrations are designed to engage students of senior level Control Systems Design course in actual engineering problems to develop their practical engineering skills and to enhance students' connection with junior level courses such as microcontroller programming. By utilizing the available resources within the College of Engineering at CSU Chico, multiple projects were developed for the Control Systems Design course. These projects benefited from existing laboratory spaces and equipment, which included a wind tunnel and a solar photovoltaic array at the Energy Systems Lab and a water flume at the Fluid and Mechanics Lab. The control systems were implemented using either the TM4C123GH6PM, the Tiva ${ }^{\mathrm{TM}} \mathrm{C}$ Series microprocessor, which students use in the two required embedded systems courses in the curriculum, or an Arduino microcontroller.

Prior to the assignment of the projects, the properties of several control algorithms were discussed during the course lectures. Hands-on demonstrations of the algorithms were performed using a Quanser QUBE Servo 2 inverted pendulum. To continue this learning, students were asked to form small teams and to select a project from a list provided by the course instructor. Examples of three projects will be described in this paper. In the first project, a controller was designed to optimize the performance of the wind turbine by maximizing the power delivered to the wind turbine load. The second project was an optimization of performance of a motorized solar photovoltaic panel where students designed a sun tracker, a proportional-integral-derivative (PID) controller to maximize the power generated by the solar panel by adjusting the angle of the panel with respect to the solar irradiance. The third project was a control system to fix the lateral position of a boat in the water flume while it was navigating along the flume to prevent contacts with the walls. Finally, a discussion on how the set of demonstrations and the design projects boosted students' interest in the Control Systems Design course. An assessment has been conducted to evaluate the impact of the demonstrations, labs and project on the student learning outcomes and the depth of their understanding of key concepts in comparison to that obtained in the previous semesters. The assessment results show that the inclusion of the demonstrations, labs and design projects in the course resulted in increased mastery of control theory concepts through the visualization and hands-on experience and that students were better able to relate theory to practice.

\section{Introduction}

The Control Systems Design is a required senior-level course in the Electrical/Electronic Engineering curriculum, offered by the CSU Chico EECE Department. The learning outcomes in this course include developing mathematical models of control components and systems, simplifying block diagrams, calculating transfer functions and state space models of linear, time- 
invariant systems, and formulating and/or utilizing specifications needed to design system controllers [1]. Therefore, the course lectures include a significant amount of time presenting mathematical derivations and discussing control theory. Students can easily lose their interest in abstract concepts such as those covered in this course and, consequently, their understanding of the course material will not reach the desired level by the end of the course. Active learning has been shown to improve student engagement and increase the depth of theoretical knowledge. Therefore, including hands-on activities would be beneficial in resolving such issues [2,3]. An additional motivation to include hands-on projects and demonstrations in our control system design course is that these activities support the attainment of ABET student outcome (6): an ability to develop and conduct appropriate experimentation, analyze and interpret data, and use engineering judgment to draw conclusions [formerly student outcome (b)] [4].

In many of the universities, courses similar to our control system design course do not include laboratories. Likely reasons for this is the cost to purchase, maintain, and upgrade experimental apparatus; facilities requirements that can include compressed air and/or hydraulics in addition to electric power; and the lab space to house the traditional control system equipment, which can have a fairly sizable footprint. Given the low demand, there are a limited number of vendors who sell control system lab equipment and, hence, the range of apparatus that is available is also limited. This leaves faculty with very few options when designing demonstrations and hands-on activities. Therefore, establishing a semester-long lab experiment for 30 40 enrolled students may not be possible when resources are limited. The authors have made multiple efforts to remove these obstacles to improved students' learning by leveraging available resources within the College, significantly lowering the cost associated with an experimental component for the control system course. Similar resources are likely available at other universities so adoption of our approach is a viable approach to introduce an experiential learning component into control system design courses offered elsewhere.

In this paper, we describe the strategies to improve student learning in this important path by employing a customized version of experiential learning model. Kolb's Model of Experiential Learning [5] relies on the humanistic perspective that experience plays a critical role in learning. The four stages of the experiential learning cycle are shown in Figure 1a. The first stage in this model is concrete experience where a student or a team is assigned a task and learn by doing, not only by watching or by listening to an instructor. The reflective observation is the step that the learner reflects on the subject by communicating with the team or another individual. The abstract conceptualization involves interpreting the experiment results. The final stage, active experimentation, is when the learner uses the outcomes of the last three steps into practice. We intend to explain how the customized version of experiential model was implemented in the class, and how it affected students' interest, depth of learning, and attainment of course outcomes compared that obtained in a semester when this model was not implemented. Kolb's Experiential Learning Model implementation starts by concrete experience whereas our learning model starts with knowledge formation meaning that students attend the lecture sessions and understand the theories. Therefore, our experiential learning model, shown schematically in Figure 1b, is a customized version of Kolb’s Experiential Learning Model. 
As the Control System Design course is heavily based on calculus-level mathematics, it would not be possible to follow the concepts only through the experiments, particularly if the system contains a nonlinear feedback response. Students first need to learn the theories and the application of math to achieve control of a complex system (Knowledge Formation step). For example, students must convert system differential equations into transfer functions for inclusion in their simulation, perform stability analysis, and learn the process steps in control design before they have the tools needed to engage meaningfully in a reflection about a control system design. Therefore, the first three steps of Kolb's Experiential Learning Model were implemented approximately midway through the semester after lectures on these topics had been presented and students had submitted several homework assignments on the material. Once they had exposure to the theories and had gained some experience applying the theories to abstract problems, students were asked to operate the QUARC controller, share the results and analyze the results from the experiments (Concrete Experience, Reflective observation, and Abstract Conceptualization), as will be explained in section II. The last step of Kolb's Experiential Learning Model (Active Experimentation) was implemented when the students obtained more information about the project (another Knowledge Formation) and worked on their final projects in which they designed a system and its PID controller from scratch, applying their knowledge and experience obtained in the prior steps and reinforced during the remaining class lectures. The results of the projects were summarized by the students in a formal report, providing a final opportunity for students for reflection (another Reflective Observation step). This is explained in more detail in section III of the paper.
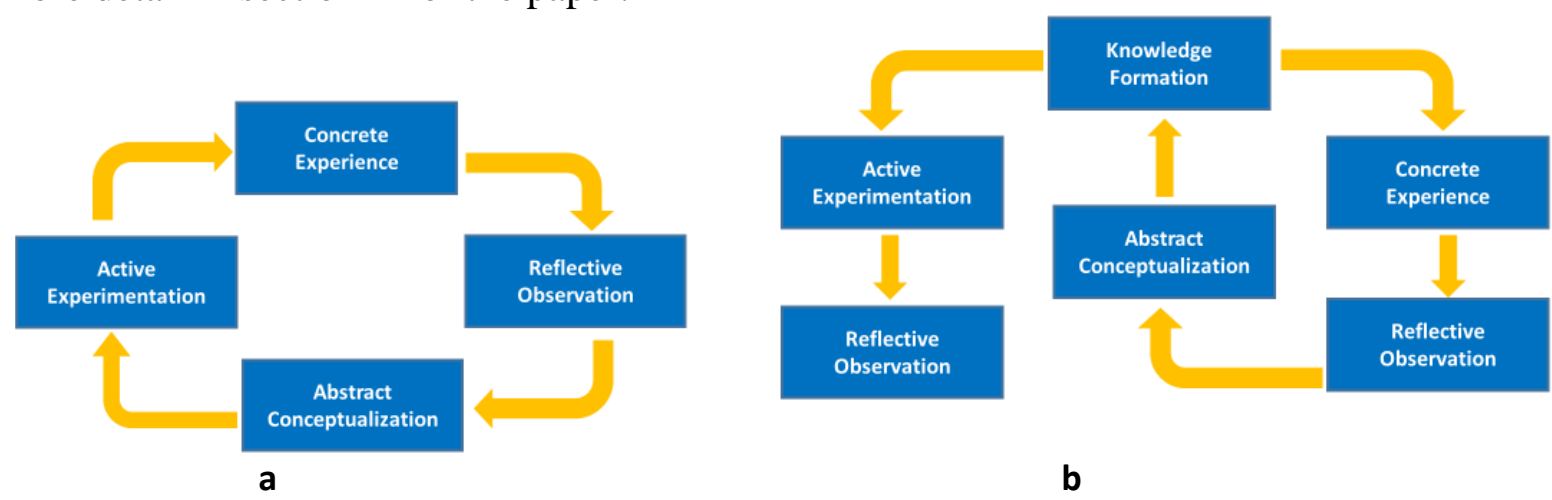

Figure 1: a) Kolb's Experiential Learning Cycle [6] and b) the customized cycle employed in this study

\section{Using the Quanser QUBE-Servo}

To address the needs for demonstrations in the control system design course, it was decided to purchase QUBE-SERVO workstations, produced by Quanser Consulting Inc., as part of a funded a CSU Chico student learning fee proposal. Given the expense, it was not possible to purchase a suite of workstations in sufficient number to enable small teams of students in a class of thirty five to use the equipment. Therefore, three sets of the equipment were purchased, and the equipment were used in demonstrations of various control theories in six sessions. Students, in groups of 3, sat at the stations while the course instructor explained the simulation models. The 
students ran MATLAB simulations. They operated the QUARC controller (Concrete Experience of Kolb's Learning Model). The students then shared their understanding with the rest of the class (Reflective Observation of Kolb's Learning Model) and analyzed the results from the experiment, altering system and controller parameters (Abstract Conceptualization of Kolb's Learning Model). The students would then change their seat with the rest of the group at each workstation who had only seen the simulations and their results on the projector screen, so that each group had an opportunity to run the system and collect their own data.

The demonstrations and experiments that were conducted in the class include the following:

1- Familiarize themselves with the Quanser QUBE-Servo hardware and the QUARC software that is used to interact with QUBE-Servo system

2- Use an encoder to measure speed, and filtering to remove noise from the speed measurements

3- Stability analysis through measuring position and speed

4- Create and validate a system first-order model

5- Create and validate a system second-order model

6- Proportional-derivative (PD) and proportional velocity (PV) control of an inertia disc

7- Modeling of the rotary pendulum

8- Rotary pendulum balance control through proportional-integral-derivative (PID) control

The experimental workstation for two of the experiments are shown in Figures 2 and 3. These experiments were demonstrated in the class to provide students with a better understanding a servo motor, proportional velocity (PV) control of an inertia disc, and PID-based balance control of rotary inverted pendulum [7]. The servo motor and inertia disc can be seen in the lower right of each figure; the inverted pendulum arm is shown only in Figure 3. In the first experiment, students learn to control the maximum peak overshoot and the speed of the response by inserting a PV controller, which is a variation of a proportional-derivative (PD) controller (Fig. 2). In the second experiment, students learn to balance the pendulum in the upright position while maintaining a desired position of the arm (Fig. 3).

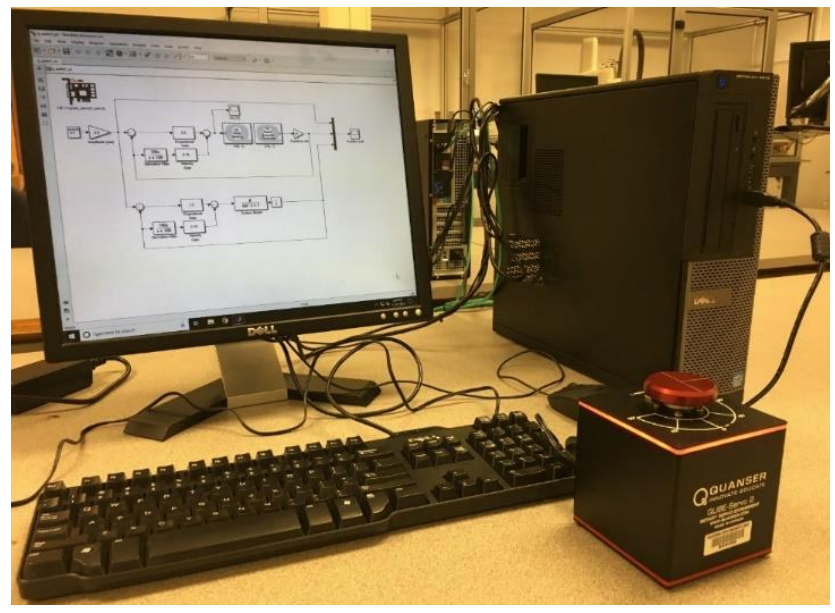

Figure 2: Block diagram of a PV control of an inertia disc and the experimental apparatus 


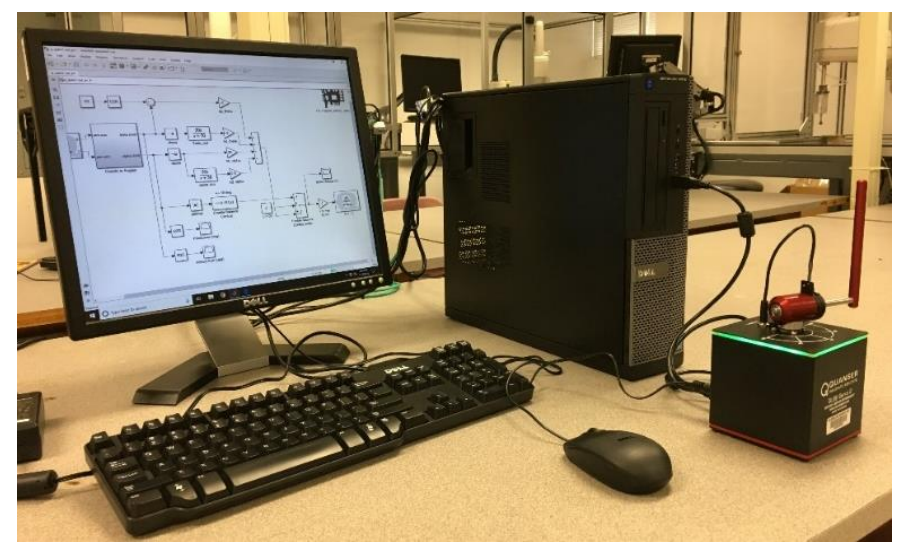

Figure 3: Block diagram of a PID-based balance control of rotary inverted pendulum

Figure 4 shows the Simulink schematic of the PV control of an inertia disc, which included the actual system (hardware-in- the-loop (HIL)) and the servo system connected to an inertia disc using a first-order model. Proportional gain and velocity gain have been considered in both the actual system and the model simulation to improve the position output in terms of speed and maximum peak overshoot.

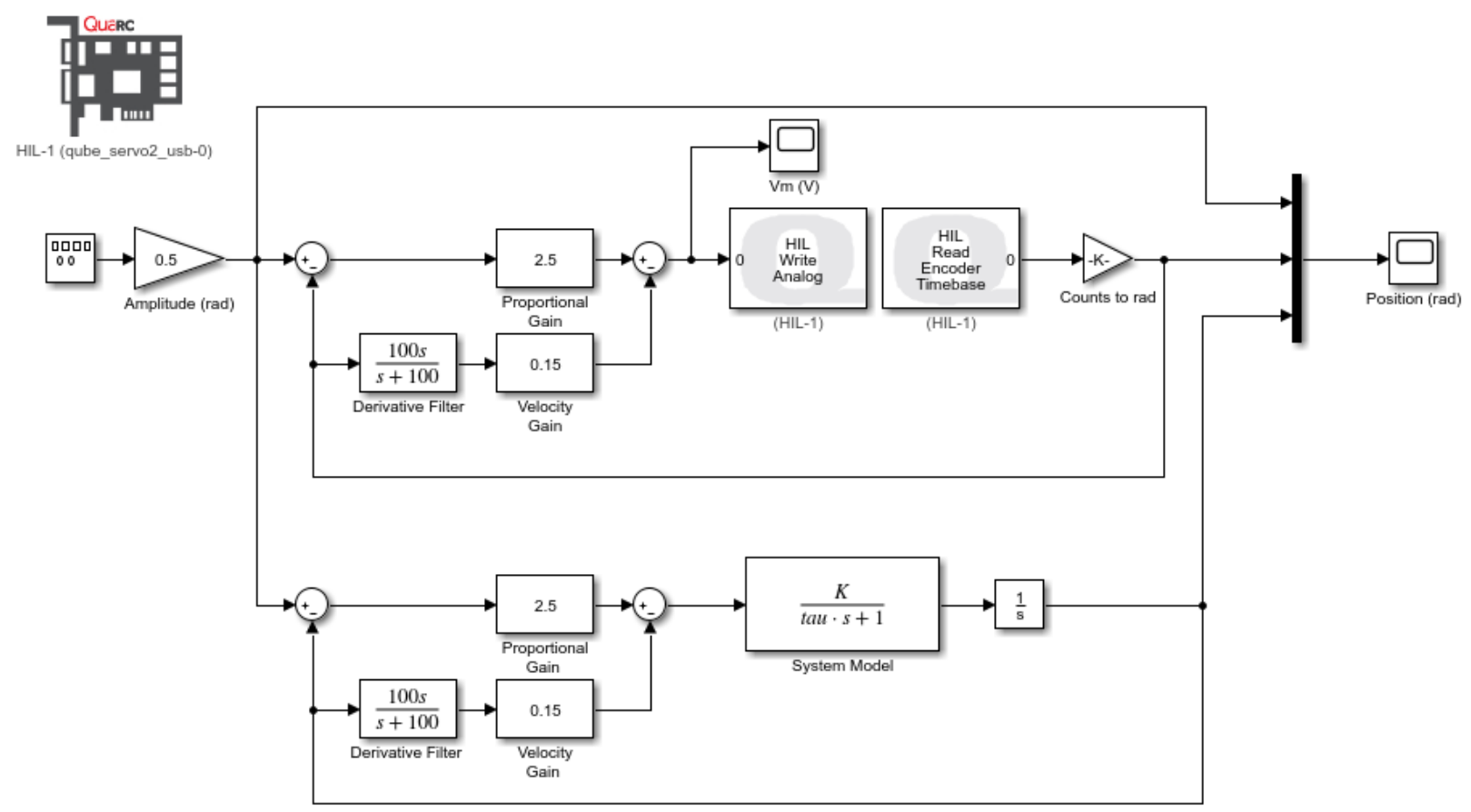

Figure 4: Schematic of PV control of an inertia disc [7]

Figure 5(a) shows the controlled position when the proportional gain was 2.5 and the velocity gain was 0.15 . The low pass filter $\frac{100}{s+100}$ was used to eliminate high frequency noise. The red 
curve is a plot of the output of the actual system controlled by the PV controller. This output had negligible overshoot and was faster than the response of an uncontrolled system (Fig. 5(b)). The response of the controlled and uncontrolled systems are compared in Table 1.

Table 1: Comparison of performance characteristics of the QUBE Servo system with and without the PV controller

\begin{tabular}{|c|c|c|c|}
\hline Servo and the inertia disc & Maximum peak overshoot & $\begin{array}{c}\text { Rise time }(\mathrm{sec}) \\
0 \%-100 \%\end{array}$ & $\begin{array}{c}\text { Settling time (sec) } \\
2 \% \text { criterion }\end{array}$ \\
\hline With PV control & $2 \%$ & 0.17 & 0.17 \\
\hline Without PV control & $70 \%$ & 0.08 & 1.5 \\
\hline
\end{tabular}

Figure 6 shows the Simulink schematic of the PID based balance control of rotary inverted pendulum when employing a PD controller. The Enable Balance Control switch was used since the designed control worked when the inverted pendulum was in a small angle range from the upright position $\left(10^{\circ}\right)$. The 0 constant that was the input to the summing point was the reference input for the rotary arm, which was set at the desired upright position. The inverted
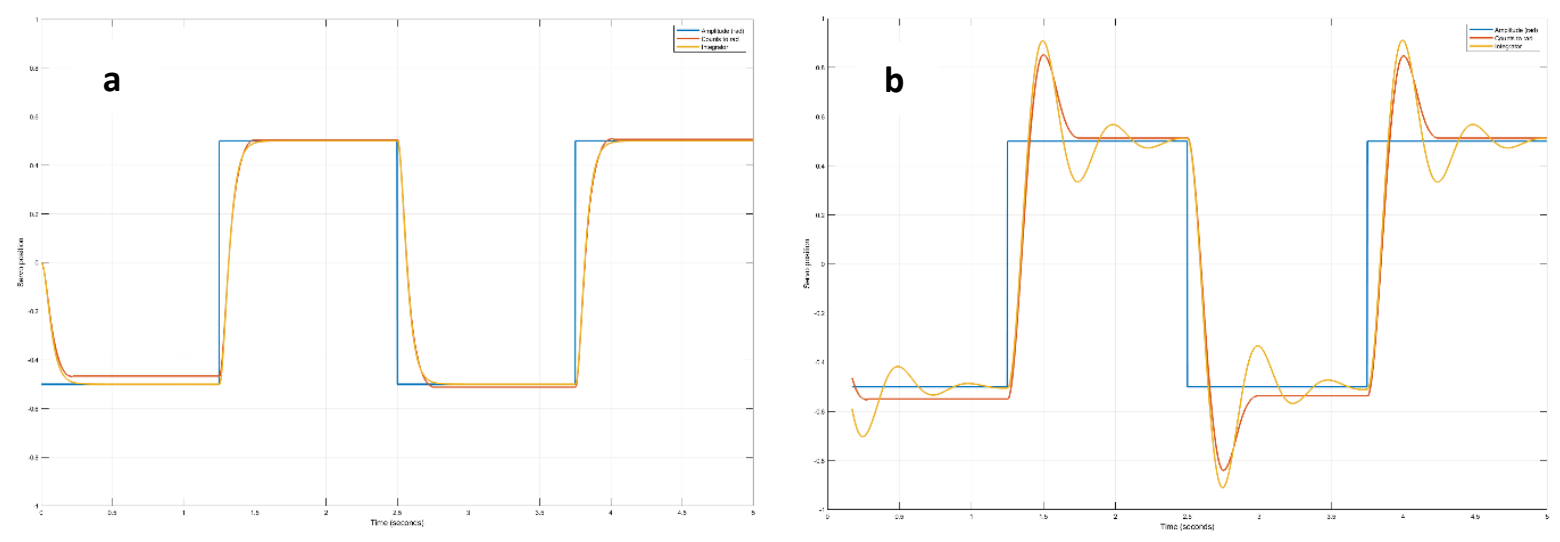

Figure 5: a: Controlled response of the QUBE servo and inertia disc actual system (red) and the system first-order model (orange) to the reference input (blue square wave). b: The response of the same system without the PV controller. Colors in Fig 5(b) are defined as in Fig. 5(a) 


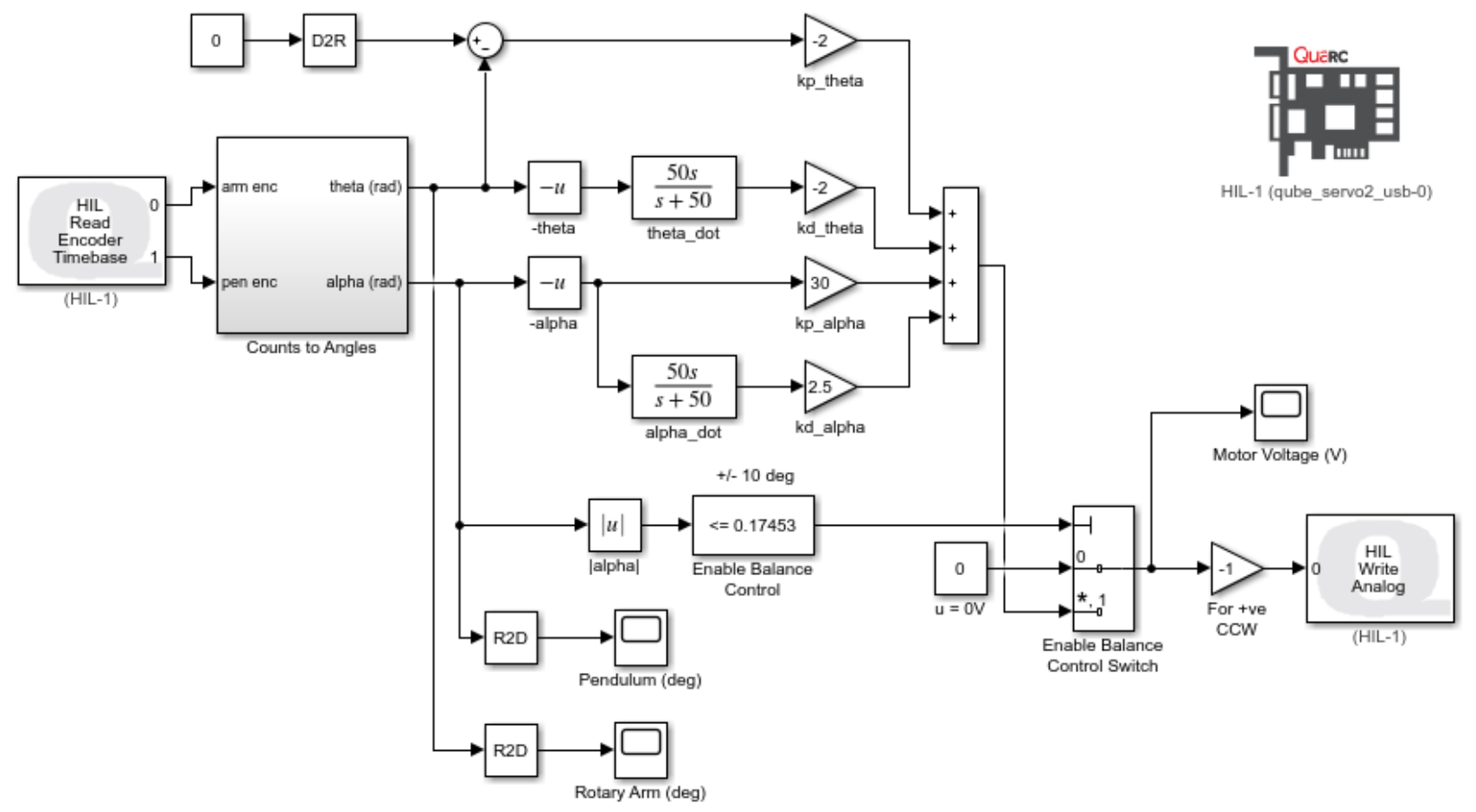

Figure 6: Schematic of PID based balance control of rotary inverted pendulum [7]
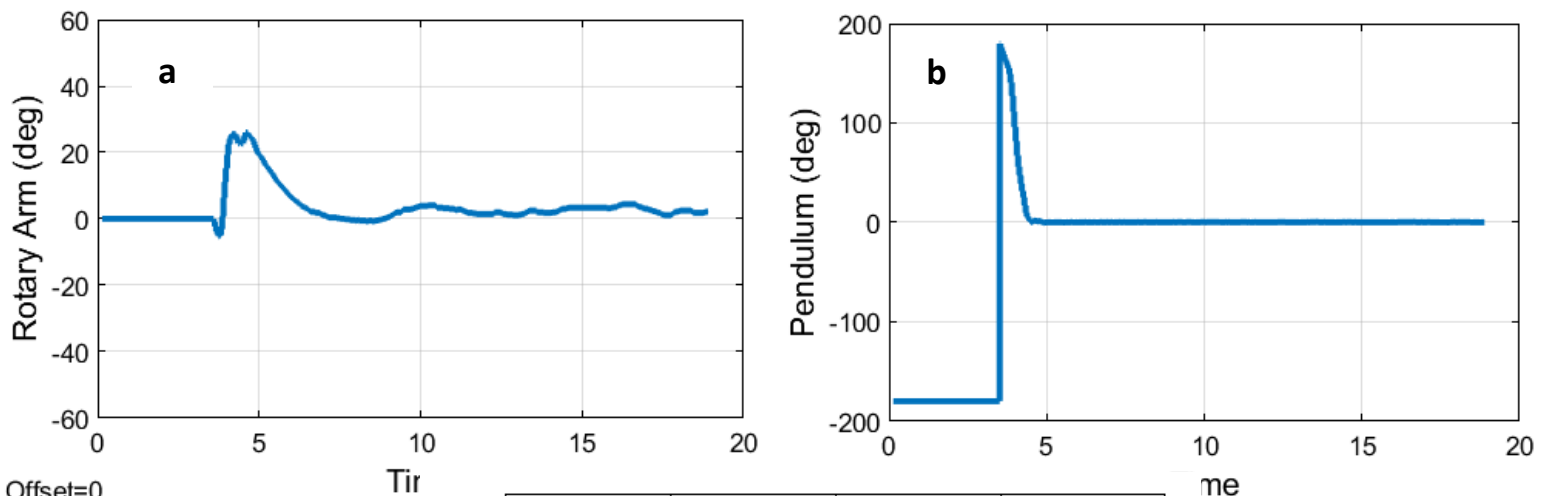

Offset $=0$

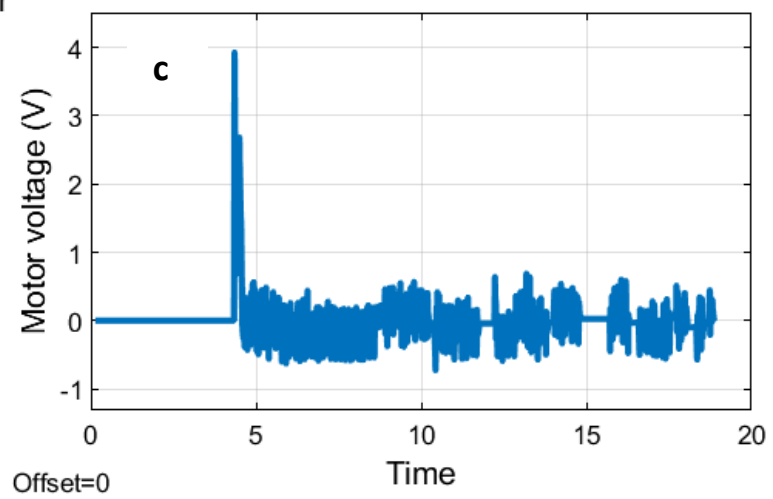

Figure 7: System response of the a: controlled rotary arm position, b: the position of the controlled rotary inverted pendulum and c: the motor voltage 
pendulum final position must be upright if the PD controller was ideally controlling its location. Figure 7(a) shows that the final rotary arm position was approximately 0 . Therefore, the control action was successful. As can be seen in Figure 7(b), the rotary pendulum final position was 0 , which resulted in 0 steady state error. Students observed the effect of four proportional and derivative gains and compared their ability to decrease the overshoot, oscillations, and steady state error and to increase the system's speed of response. Also, students gained an appreciation that a linear controller worked over a limited range with nonlinear systems. The nonlinear systems and linearization techniques around operating points had already been discussed during the lectures prior to the demonstration. Therefore, students had the necessary background of the topic to draw the desired conclusions after observing the operation of the system.

By observing these effects and analyzing the contribution of the experimental parameters, students went beyond the traditional theory, calculation, and simulation to practical implementation. Students learned better through visualization and hands-on experience, and will be able to relate theory to practice, which made them more confident in practical design challenges presented in the following assignment, the control system design project.

The demonstrations and experiments by Quanser QUBE-Servo served as great tools for the first three phases of the experiential model after the theoretical concepts were discussed in lectures. Students obtained concrete experience and were asked to make reflective observations and described their abstract conceptualizations. This proved to be an effective way to prepare students for their design projects, which required that they would once more go through all four phases of experiential learning cycle.

\section{Examples of the final projects}

As active experimentation is an important part of the Experiential Learning Model, final projects were assigned to the students. In these projects, students were expected to apply what they previously learned from lectures, labs, and demonstrations in projects, which they conducted individually or in small teams (2-4 students). The topics of the projects were selected by the students. Multiple ideas for projects were proposed by the instructor, which also served to inform the students about the resources available in the college. Some students selected the suggested projects and others proposed their own. Amongst these topics, as stated earlier, were PID control of the position of a motorized boat in a water flume, design of a controlled motorized sun tracker for a solar panel, and PID control of a wind turbine to maximize the power delivered to the load. The following discussion is an overview of each of these three projects.

\section{A. Controlling the boat trajectory in a water flume}

The objective of this project was to construct a motorized boat, fabricated by 3D printing, and to design its control board. The control board would guide the boat as it moved the length of the water flume and to correct its position laterally when drifting in the water. Two ultrasonic sensors (HC-SR04) were used to sense the proximity of the obstacle (the flume walls). The 
signals from the sensors were read by an Arduino microcontroller, on which ran the control algorithm. The output from the algorithm was used to determine the power delivered to each of the boat's two motors and, thus, to divert the boat away from the obstacle and to continue travelling forward. Each proximity sensor emitted a series of $40 \mathrm{kHz}$ ultrasonic pulses and produced a signal that was proportional to the time it took for the pulses to return to the sensor. The measured time of the signal round trip from a particular sensor determined the distance of the associated motor from a flume wall.

The motors were powered by an external battery. The speed and direction of rotation of each motor was determined by the operation of an L298N H-bridge, which was controlled by a pulsewidth modulation (PWM) signal from the microcontroller. The PWM signal was determined by the control algorithm run on the microprocessor, using data from the proximity sensor data as the input. The student-constructed boat and circuitry are shown in Figure 8.

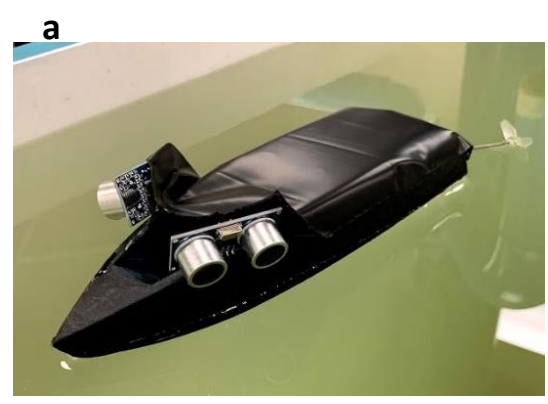

b

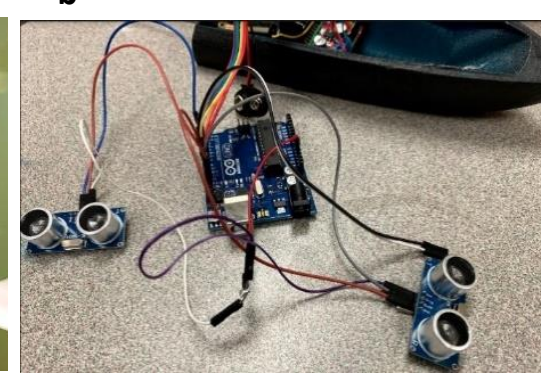

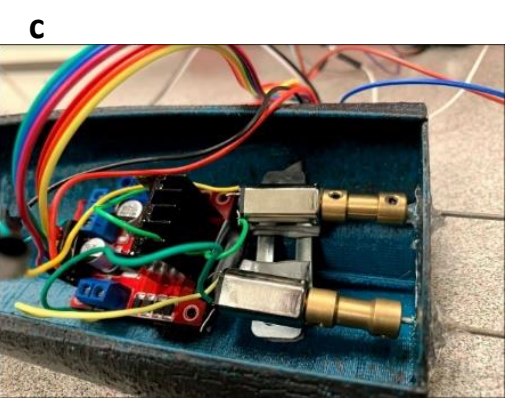

Figure 8: The boat after assembly, b: sensors and microprocessor, and c: H-bridge and motors

Figure 9 is the control circuit configuration used for the boat trajectory control. The students determined that a PID controller was required to achieve the necessary performance characteristics, which included an analysis of the overshoot, speed, and steady state error. The control board kept the boat in the center of the path by not allowing the measured distance from one of the motors to the wall to become more than the distance from the other motor to the opposite wall.

To design a PID controller for the system, system characterization was carried out by applying a step input to the actual system and measuring the response. The performance characteristics such as maximum peak overshoot, rise time, and settling time were calculated from the step response to obtain the damping ratio and natural frequency and model the system as a second-order system. The students determined that the PID controller had to be designed to meet the desired system response in terms of maximum peak overshoot, settling time and oscillations. In the next step, the PID controller proportional, derivative and integral gains were found. The students had learned multiple ways to determine these gains from the demonstrations conducted previously in class, which included using the Matlab PID tuning toolbox and the Ziegler-Nichols method [8]. The PID controller was implemented in the Arduino microcontroller to improve the performance of the system. 


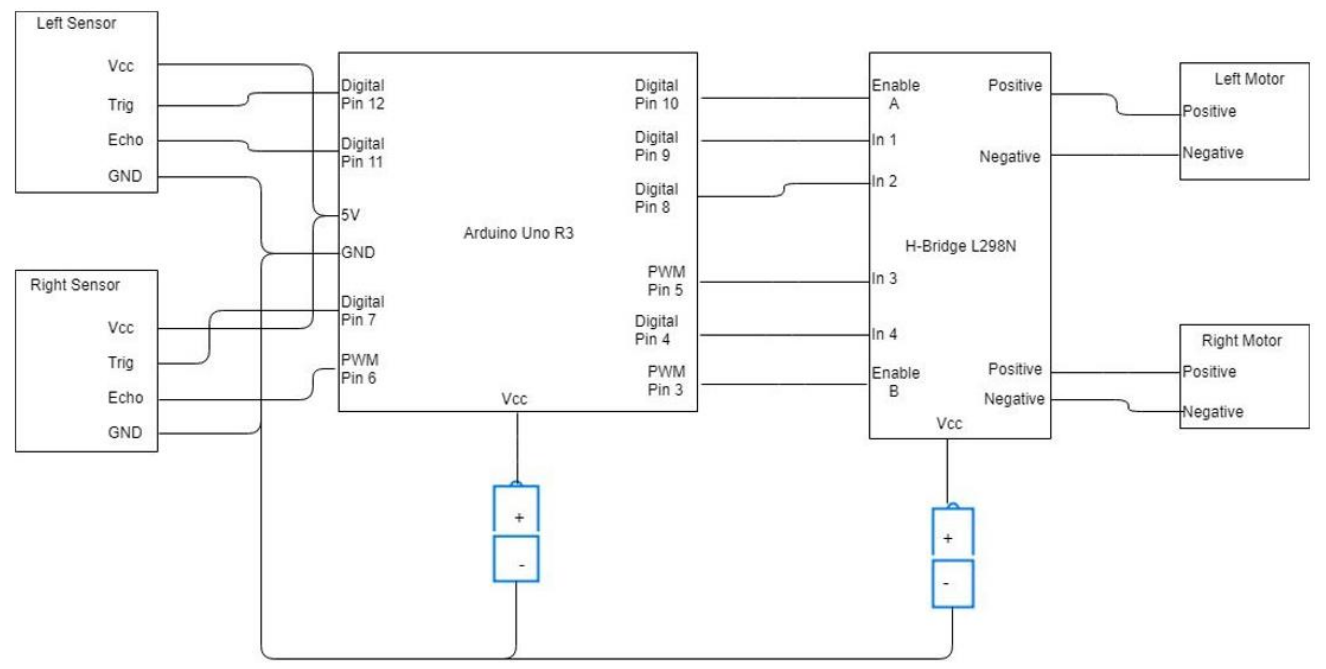

Figure 9: Control circuit configuration for the boat.

The students encountered several challenges in this project. The largest problem was the friction of the motor shaft connectors with the rods of the propellers. This issue was better addressed when testing the boat in the water as the water placed a considerably different load on the motors than did air. This friction caused the motor to run slowly when the boat was in the flume. Therefore the gains of the PID controller, determined when the motors were working against air, did not produce adequate control of the boat's location in the flume. Secondly, the project definition required that the system be limited to footprints no larger than $12 \times 12$ inches ${ }^{2}$. Given the weight of the sensors, motors, microcontroller, and other electronics, fitting most of the hardware in the interior of the boat while maintaining enough buoyancy to keep the boat float presented significant difficulties to the team. Access to the water flume for experimentation had to be arranged around the Civil Engineering's lab schedule, which did not present a significant barrier to the students as they worked on the project. Overall, students managed to perform the required tasks to complete this project and attain the course outcomes by means of the implemented experiential learning model.

\section{B. Position control of a sun tracker}

To maximize the power generation from a solar panel, the position of the panel must track the position of the sun, ideally in both azimuth and altitude. A control circuit can be designed using one or more light sensors and a microcontroller to command a motor to rotate the solar panel to the optimal location. Four light-dependent resistors (LDR) were employed to measure the intensity of sunlight, at one corner of the solar panel. One servomotor was used to move the solar panel to rotate the panel from an azimuth of $+/-1$ angles. The control algorithm was run on an Arduino microcontroller, which output the needed PWM signals to drive the servomotors, based upon the inputs collected from the LDRs. When the readings from all four LDRs were equal within an allowed tolerance, the panel would collect the highest intensity of sunlight. Figure 10 shows a simple architectural design of the control system of a sun tracker designed by single student responsible for the project. 


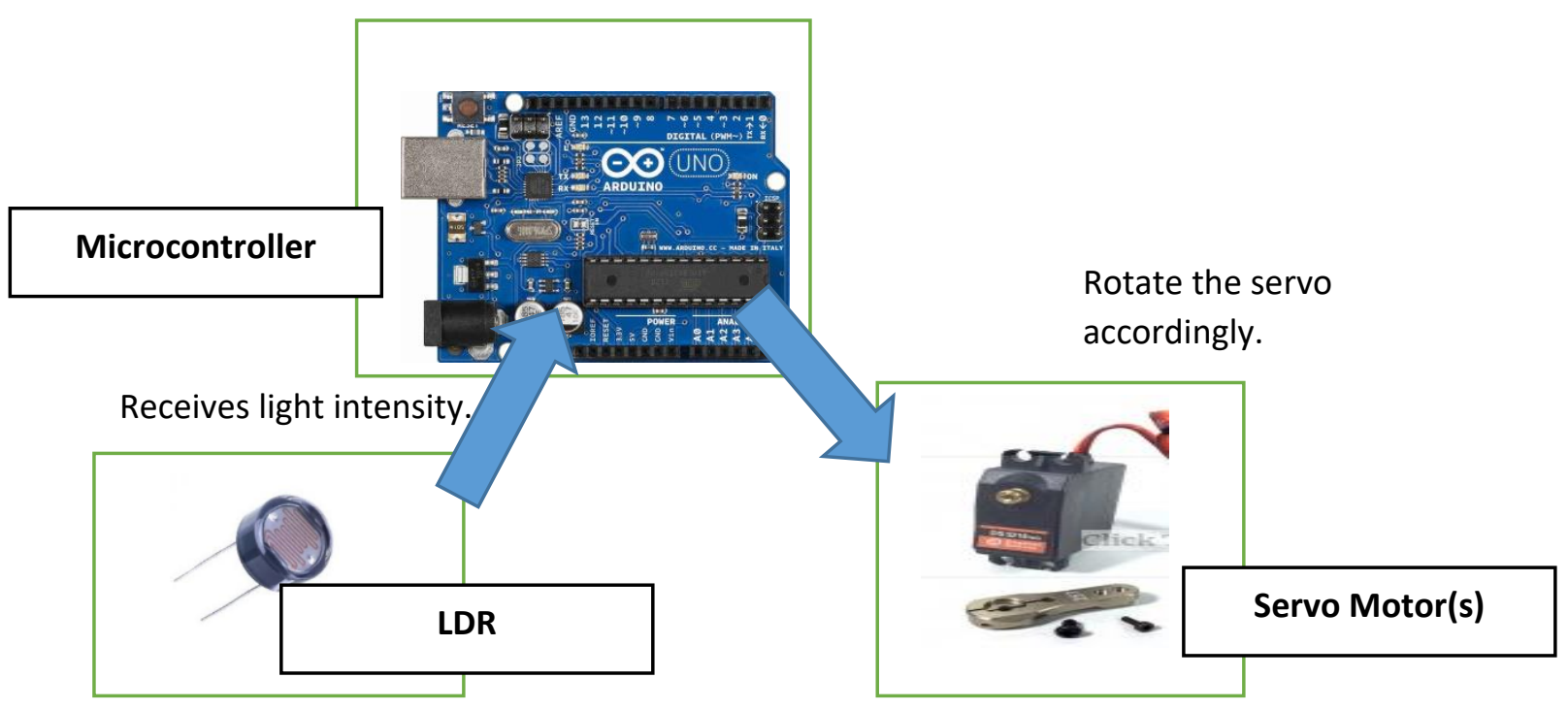

Figure 10: The architectural design of a sun tracker control system

Figure 11 demonstrates the actual solar panel that the student used and the circuitry descripted in Figure 10. Two nontechnical challenges associated with this project were that the control panel of the existing two-axis controlled solar panel could not be modified and that the solar panel was needed at several times during the week for experiments in another course by the time the student was ready to begin the experimental phase of his project. Therefore, the student chose to work with a smaller solar panel for which the control system could be designed from scratch and could be moved outside the lab, enabling the student to work on the project at any time.

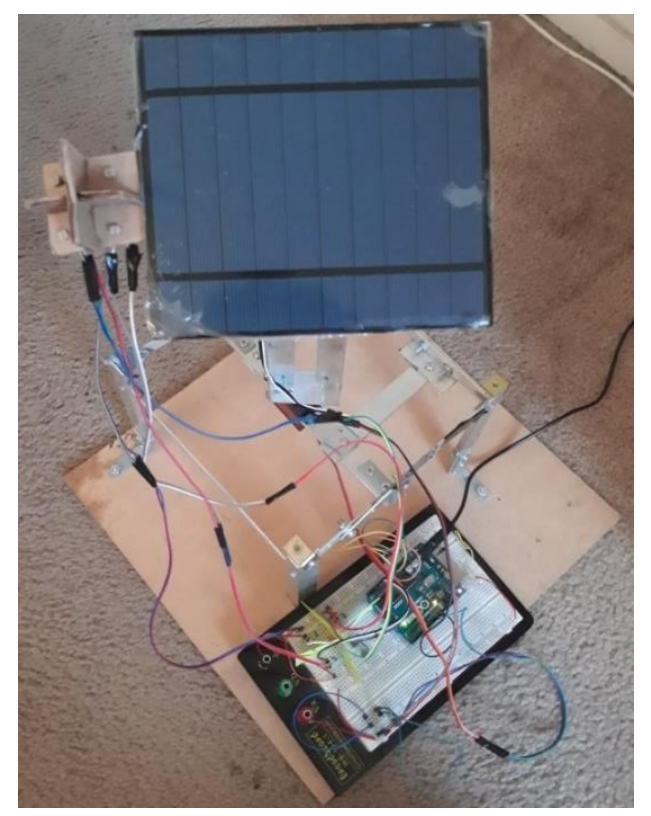

Figure 11: The solar panel and control circuitry 


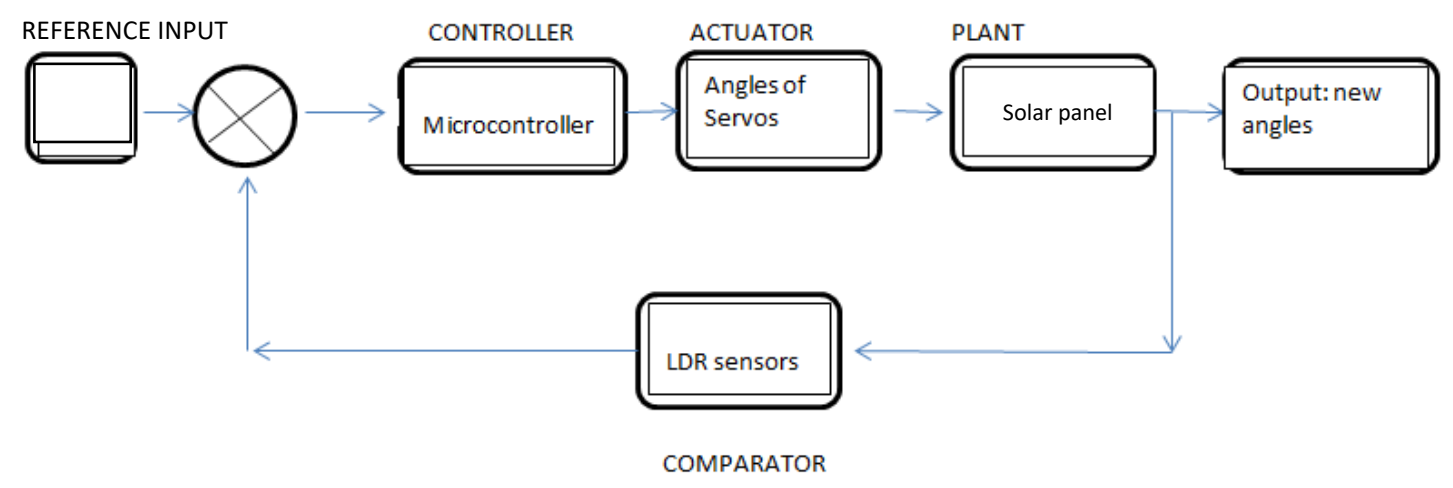

Figure 12: The block diagram of a sun tracker control system

The servomotor used in the sun tracker was found to have satisfactory performance in terms of steady state error. To adjust the speed of the response, a PID controller was programmed in the Arduino microcontroller. The design process steps were, again, to first use the step response to obtain system performance characteristics such as time constant for a first-order system model, and secondly to determine the maximum peak overshoot, rise time, and settling time for a second-order system model. Then, the controller could be designed using the characterized system transfer function. The block diagram in Figure 12 is the control system of the sun tracker with feedback from the LDR sensors. The reference input was the initial position of the solar panel. Overall, the project served as an important tool in honing student's hands on experience, connecting between theory and practice, and understanding different elements of a control system.

\section{Maximizing the power delivered by a wind turbine to a load}

The purpose of the third project was to design a PID controller to maximize the output power of a wind turbine generator that was delivered to a load. The first step was to characterize the system, which required an analysis to find the trend of the power delivered to the load as the turbine was rotating inside the wind tunnel as a function of the angle of the turbine blades to the direction of wind. The control panel of the wind tunnel did not allow students to control the speed of the wind in the tunnel. Therefore, the speed of the turbine blades as a function of position of the turbine was the only input to the control system and the position was determined when the student placed the turbine in the wind tunnel. The load was a variable resistor, the value of which could be changed by a servo motor. Thus, the value of the resistance of the load could be adjusted as the power generated by the turbine changed to obtain the possible maximum power dissipated by the variable resistor at any point in time. The microcontroller was used to control the direction of the servomotor rotation. The power dissipated by the load resistor was calculated using square of the measured voltage drop across the resistor divided by its resistance, determined from a lookup table that correlated resistance with angle of the servomotor. The block diagram of the feedback system is shown in Figure 13. 


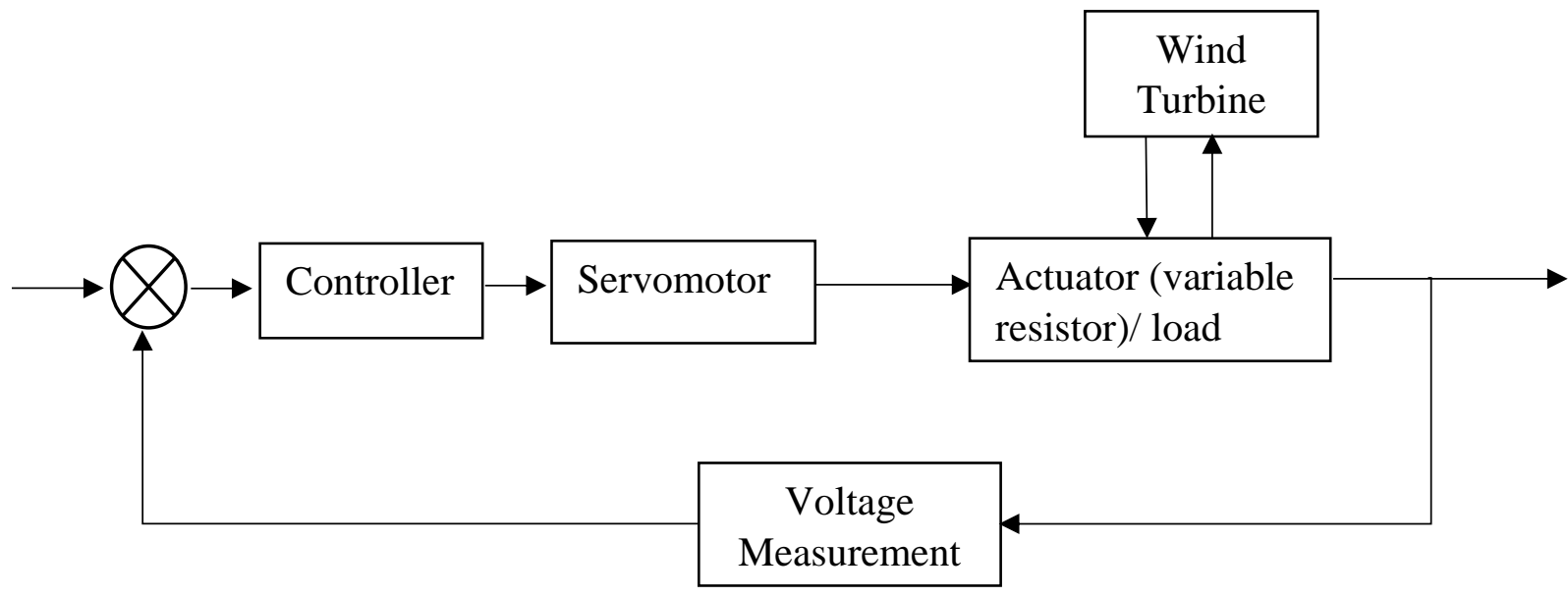

Figure 13: The block diagram of wind turbine connected to the variable resistor that is being controlled by a servomotor.

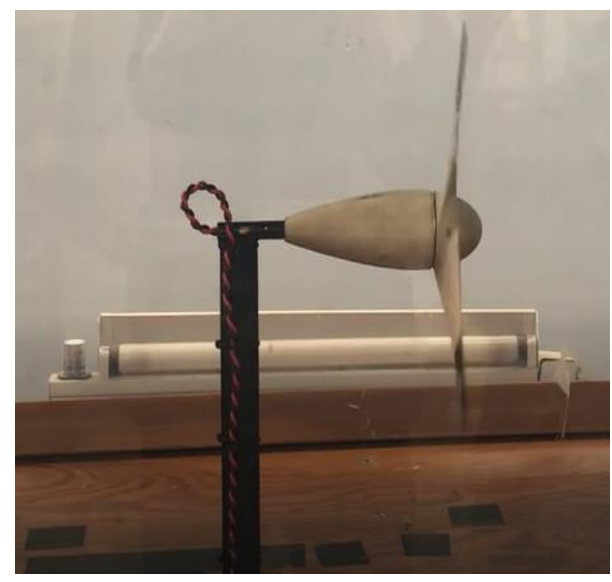

Figure 14: Wind turbine inside the wind tunnel

The system was characterized by applying a fixed speed wind to the turbine and reading the load voltage across the variable resistor, which determined the delivered output power. The system output (delivered power) versus time was a first-order curve. After characterization of the system, the students designed a proportional-integral (PI) controller, and observed that the rise time is significantly less than that of the uncontrolled system. Any derivative control seemed to have negligible effects in the simulations; thus to decrease unnecessary complexity, derivative control was not used. One of the difficulties of this project was the fact that our electrical engineering students were not familiar with the operation of the wind tunnel. Because of this and other safety concerns, the students could only work under the direct supervision of the faculty member who was responsible for the wind tunnel. Thus, the students' progress on the project was slower than expected. 


\section{Assessment of student success}

The inclusion of the lab demonstrations and final projects in Fall 2018 had a significant positive impact on the average final grade in the Control System Design course compared to the course average in Fall 2017. The class average increased from 72.81/100 in Fall 2017 to 83.79/100 in Fall 2018, a 10.98\% improvement. This alone shows that the experiential model has been effective. Another indication of the effectiveness of the experiential model is that $94.3 \%$ of the students passed the course in Fall 2018. Whereas in Fall 2017, 89.47\% of the students passed the Control System design course, a $4.83 \%$ improvement. The depth of learning was assessed by their performance as students designed different types of controllers in their projects and exams. In Fall 2018, 74\% of the students solved the control design problems on the final exam completely correctly. Whereas in Fall 2017, only $48 \%$ of the students solved the problems correctly. Therefore, there was a $26 \%$ improvement in understanding the controller design concepts. Compared to students in Fall 2017, students who engaged in the concrete experiences and active experimentation were more confident in their abilities to design control systems. In Fall 2018, far fewer students came to office hours for assistance and they demonstrated a better theoretical understanding when they asked questions during the class lectures and office hours. Additionally, they were more confident in designing lead and lag controllers after they had observed demonstrations and simulations, conducted experiments during the labs and designed and tuned PID controllers during the course of their projects. This was clearly observable from the questions they asked during the class sessions and office hours.

As this required course is only offered in the Fall semester and no equivalent course taught in the Spring semester has been identified, graduation with a BS in Electrical/Electronic Engineering is delayed a year if a senior student does not pass our course. Therefore, applying teaching strategies that promote student learning is essential, not only to encourage a deeper understanding of the concepts but also because of the financial impact that failure to learn the material to a minimum satisfactory level will have on the student. Thus, the introduction of the concrete experiences and the active experimentation into the Control System Design was a success as a graduation initiative as the failure rate in the course was halved.

Several issues were identified after a review of the student projects at the end of the semester. First, some students did not manage their time well and had difficulties finishing the planned tasks. While students were informed about the project assignment at the beginning of the semester, many students did not focus their efforts on the project until after the Thanksgiving holiday was over, leaving little time to develop and implement a design, let alone address any unexpected obstacles. A useful step to motivate students to make timely progress on the project is to set intermediate deadlines, such as a midterm report, rather than a single deadline when the students must submit the final project report. Recent lead-times for some electronic components have been increasing, which delayed their receipt, and must be considered in the project planning. Another intermediate report is to set a deadline for students to obtain reimbursement from the department for components that students order. As noted in the description of the three projects in this paper, students were using outside department's equipment and facilities. As the equipment and facilities were also needed for labs held by the other departments, the students in 
the Control System Design course had to coordinate times to use the equipment and facilities around the other departments' lab schedules. Some of the projects also could not conduct experiments using the equipment and facilities without the direct supervision of certain faculty and staff. This compromised the time for experimentation available to our students, particularly those students who worked or commuted to school. Therefore, requiring students to acknowledge this limitation and factor it into their project schedule will have to be considered.

\section{Summary}

A customized version of Kolb's Experiential Learning Model was implemented successfully in the Control System Design course. The addition of Quanser QUBE equipment to the course sessions as demos and labs boosted students' interest and eagerness to study during the semester. Control System Design is the first course that students use the Simulink intensively. Having these demos engaged students more in Simulink, and increased their ability to apply it in their final projects. Moreover, students learned how to implement a controller and visualized how the response of an actual system is improved employing controllers. The final projects enhanced students' hands on ability and helped them to apply their learnings in demos/labs and lecture sessions. It was also observed that students became strongly engaged in the course while working on their final projects. A higher level of comprehension of control theory was observed by the course instructor when interacting with students in class and individually. Student performance in the course was improved and a higher percentage of students completed the course successfully in the semester in which the experiments and projects were introduced.

\section{Acknowledgement}

The authors would like to thank the students Khang Yang, Ray Angelo Siapno, Luis Cortez, Jose Cerna, Edward Bonilla, Bryan Gentles, Diana Garcia, and Sandy Sam who worked on the three projects descripted in this paper. Dr. David Alexander and Dr. Steffen Mehl, faculty members in the Department of Mechanical and Mechatronic Engineering and Sustainable Manufacturing and the Department of Civil Engineering Department, respectively, graciously volunteered their time to support the final projects and supervise the students when using their departments' lab facilities. The three projects described in this paper could not have been completed without their assistance. Dr. Z. Alavi would also like to acknowledge the financial support from CSU Chico Student Learning Fee award, which funded the purchase of the Quansar workstations.

\section{References}

[1] Zahrasadat Alavi, Arash Kialashaki, "Towards Removing Barriers Against Learning Control Systems Design: A Comprehensive Review of the Math Required for Reaching Milestones in Control Systems Design”, 2018 ASEE Annual Conference and Exposition, Salt Lake City, Utah, June 23-27, 2018. 
[2] Nahid Uzzaman, Dale Schinstock, "Evaluation of Inexpensive Hardware for Control Theory Application", 2018 ASEE Annual Conference and Exposition, Salt Lake City, Utah, June 23-27, 2018.

[3] Curtis Cohenour, "A Low Cost Control System Experiment for Engineering Technology Students", 2017 ASEE Annual Conference and Exposition, Columbus, Ohio, June 25-28, 2017.

[4] Criterion 3. Student Outcomes, Criteria for Accrediting Engineering programs, https://www.abet.org/accreditation/accreditation-criteria/criteria-for-accrediting-engineeringprograms-2019-2020/\#GC3

[5] David A. Kolb, Experiential Learning: Experience as the Source of Learning and Development, $1^{\text {st }}$ Edition, Upper Saddle River, NJ, Prentice Hall, 1984.

[6] https://www2.le.ac.uk/departments/doctoralcollege/training/eresources/teaching/theories/kolb

[7] www.quanser.com

[8] Tim Wescott, "PID without a PhD", Embedded System Design, October 2000, pp. 86-108, Retrieved from https://www.embedded.com/design/prototyping-and-development/4211211/PIDwithout-a-PhD. 